\title{
Problems for the Cognitive-Depletion Model of Religious Interactions
}

\author{
Paulo Sousa ${ }^{a^{*}} \&$ Claire White ${ }^{a, b}$ \\ a Institute of Cognition \& Culture, Queen's University, Belfast \\ ${ }^{b}$ Department of Religious Studies, California State University, Northridge
}

Schoejdt, Sørensen, Nielbo, Xygalatas, Mitkidis and Bulbulia put forward a new cognitive model of religious interactions, which we depict in Figure one. The model stresses three common features of such interactions: the requirement to suppress emotion expression, the exposure to ritualized behavior and the presence of a charismatic authority (1). ${ }^{1}$ These features have different cognitive effects involving different sorts of cognitive-resource depletion (2). The regulation of emotion expression drives one's attention to inward phenomena, compromising one's ability to form episodic memories of the external, observable aspects of rituals. Ritualized behavior precludes the ordinary, means-end analysis of action and demands low-level action parsing. These two effects impede the interpretation of ritualized behavior or other observable aspects of rituals (3), which makes one susceptible to authoritative interpretive discourses (4), which in turn promote collective religious-symbolic interpretations (5). The presence of a charismatic authority has a third cognitive effect involving cognitive-resource depletion-lack of error monitoring concerning the evidence disconfirming an authoritative interpretation. Since a charismatic authority is an authority, it can play two roles: a conjoint one, by adding another force for the establishment of collective religious-symbolic interpretations $(4-1 \rightarrow 2 \rightarrow 5)$; an independent one, by persuading individuals to align their interpretations of a ritual with the authoritative one $(1 \rightarrow 2 \rightarrow 5)$. Thus, via cognitive-resource depletion, the three features contribute to the same effect, which constitutes their social function- to

\footnotetext{
${ }^{*}$ Corresponding author: p.sousa@qub.ac.uk.

${ }^{1}$ Numbers are related to Fig. 1.
} 
secure collective religious-symbolic interpretations. The remaining part of the model $(5 \rightarrow 6 \rightarrow 1)$ completes a social-functional approach, qua an explanation of the occurrence of the functional features (Nagel, 1961).

(6) Social Cohesion

(5) Collective Religious-Symbolic Interpretations

(4)

(3)

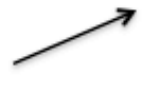
Authoritative Discourse

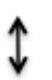
Lack of Interpretation

(2) Lack of Episodic Memory
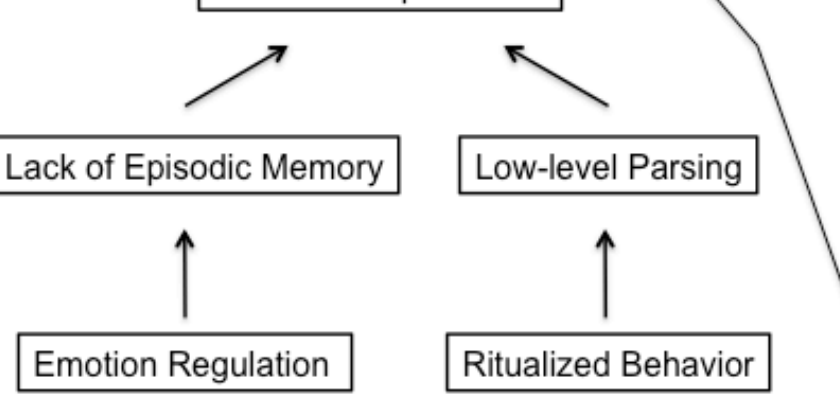

Lack of Error Monitoring

(1)
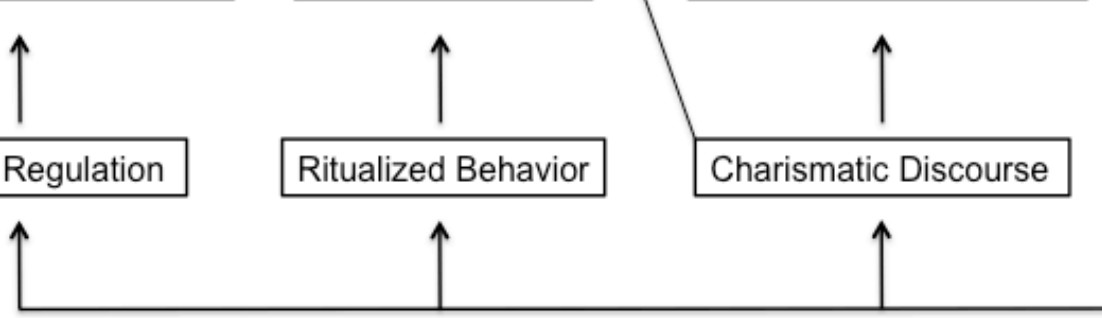

Figure 1 (Sousa \& White). Cognitive-Depletion Model of Religious Interactions. Boxes refer to causes and/or effects; arrows refer to causal relationships; sequences of boxes and arrows refer to causal chains. Numbers (and their sequences) indicate the different parts of the model. Linked boxes indicate the possibility of a composition in causal role.

In this commentary, we identify the main problems regarding causal chains $(1 \rightarrow 5)$. We do not evaluate causal chains $(5 \rightarrow 6 \rightarrow 1)$ because the authors are ambiguous about them-sometimes they seem interested in effects simply as an effect not as a function, as if eliminating these chains; sometimes they use the full language 
of social functionalism, as if supposing them.

There are two problems with the emotion-regulation causal chain. First, the preliminary evidence bears relevance to $(1 \rightarrow 2 \rightarrow 3)$, but not to $(3 \rightarrow 4 \rightarrow 5)$. The fact that two months later fire-walkers recalled, less accurately and more confidently, more external details of the ritual than they had recalled just after it, ${ }^{2}$ supposedly due to a process of authoritative social reconstruction, concerns the elimination of a memorygap in external details not the elimination of an attributional-gap in the religioussymbolic interpretation of these details. Second, it is doubtful that these findings generalize to other high arousal rituals containing the emotion-regulation feature. These rituals often involve elaborate ordeals that last a long period of time (see Whitehouse, 1996), whereas the fire-walking ritual operationalized in the field study seems comparatively simple and has a very short time span —-for each fire-walker, the ritual details to be remembered concerned simply the few seconds of the fire-walk plus the period immediately before and after it (see Xygalatas et al., submitted). Therefore, there aren't many relevant external details to attend to; unsurprisingly, the study revealed quite poor external episodic memories, both following the ritual and two months later.

There is one problem with the ritualized-behavior causal chain. The preliminary evidence speaks to $(1 \rightarrow 2)$, but not to $(2 \rightarrow 3 \rightarrow 4 \rightarrow 5)$, since the studies showing low-level parsing of unfamiliar or familiar opaque behavior did not include dependent measures directly probing interpretive cognitive-resource depletion and such depletion is not entailed by low-level parsing when interpreting others' ritualized behavior.

\footnotetext{
${ }^{2}$ It is worth noticing that the numerical difference in external-detail recall wasn't statistically significant in any of the measures of the study (see Xygalatas et al., submitted).
} 
Contrary to what is assumed by the authors, there is no similarity in terms of cognitive-resource depletion between interpreting others' ritualized behavior and interpreting one's own. Interpretive cognitive-resource depletion occurs when one's interpretive capacities cannot be utilized because one's mental resources are overloaded with other tasks. There is such depletion in interpreting one's own ritualized behavior not because one cannot interpret it in terms of an ordinary, meansend analysis of action but inasmuch as one has to carefully focus one's attention on the proper performance of its low-level units (Boyer \& Liénard, 2006). ${ }^{3}$ And the simple impossibility of interpreting others' ritualized behavior in terms of an ordinary, means-end analysis of action does not entail depletion. It does not preclude nonordinary interpretations, such as symbolic ones. It rather "calls" for them.

The authors insist that there is depletion in interpreting others' ritualized behavior because one's mental resources are overloaded with the excess of predictive error coming from parsing low-level units. However, ritualized behavior is often stereotypical and repetitive, which makes its low-level units totally predictable. In other words, the other properties of ritualized behavior overcome the predictive error related to absence of ordinary parsing and free one's mental resources to interpretation. Thus, the only interpretive problem posed by an exposure to others' ritualized behavior is the "freedom" of interpreting it in non-ordinary terms, which has nothing to do with interpretive cognitive depletion - if interpretations do not take place, it is due to absence of motivation, not to presence of depletion (coming from low-level parsing).

There are two problems with the charismatic-authority causal chains. First, the preliminary evidence is unrelated to these chains. The thrust of the authors' discussion

\footnotetext{
${ }^{3}$ The other side of the story is a "recalibration" of the motor control system and its unconscious perceptual system (Jacob \& Jeannerod, 2003; Wolpert \& Ghahramani, 2000).
} 
concerns the role of charismatic authorities in establishing collective religioussymbolic interpretations of rituals, as we characterized. However, the praying evidence they cite is about establishing a belief in the causal efficacy of a ritual, namely, in the existence of effects beyond the scope of the ritual. Second, it is doubtful that religious authorities (charismatic or otherwise) play the exact role delineated in the model—while standardizing religious discourses may guarantee a good amount of uniformity in public representations, they often do not have this effect on mental ones (Sperber, 1996).

To conclude, we applaud the authors' attempt to build a new cognitive model of religious interactions. However, more empirical support is required for their model to become a strong contender to explain such interactions.

\section{References}

Boyer, P., \& Liénard, P. (2006). Why ritualized behavior? Precaution systems and action parsing in developmental, pathological and cultural rituals. Behavioral and Brain Sciences, 29, 595-613.

Jacob, P. and Jeannerod, M. (2003). Ways of Seeing. Oxford University Press.

Nagel, E. (1961). Functionalist Explanations in the Social Sciences. In The Structure of Science. London: Routledge and Kegan Paul.

Sperber, D. (1996). Explaining Culture. Oxford: Oxford University Press. 
Whitehouse, H. (1996). Rites of terror: Emotion, metaphor and memory in Melanesian initiation cults. The Journal of the Royal Anthropological Institute, 2, 703-715.

Wolpert, D. M., \& Ghahramani, Z. (2000). Computational principles of movement neuroscience. Nature Neuroscience, 3, 1212-1217.

Xygalatas D, Schjoedt, U., Bulbulia, J., Konvalinka, I., Jegindø, E., Reddish, P., Geertz, A.W., \& Roepstoff, R. (2012). Memory in a firewalking ritual (under review). 